\title{
Around the Pair Instability Valley - Massive SN Progenitors
}

\author{
Roni Waldman \\ Racah Institute of Physics, Hebrew University, Jerusalem 91904, Israel \\ email: waldman@cc.huji.ac.il
}

\begin{abstract}
The discovery of the extremely luminous supernova SN 2006gy, possibly interpreted as a pair instability supernova, renewed the interest in very massive stars. We explore the evolution of these objects, which end their life as pair instability supernovae or as core collapse supernovae with relatively massive iron cores, up to about $3 M_{\odot}$.
\end{abstract}

Keywords. stars: evolution, (stars:) supernovae: general

\section{Introduction}

The interest in the evolution of very massive stars (VMS), with masses $\gtrsim 100 M_{\odot}$, has recently been revived by the discovery of SN 2006gy - the most luminous supernova ever recorded (Smith et al. 2007). This object, having a luminosity of $\sim 10$ times that of a typical core-collapse SN (CCSN), is probably the first evidence of a pair instability SN (PISN) Woosley et al. (2007). PISN are massive stellar objects, whose evolutionary path brings their center into a region in thermodynamical phase space $\left(\rho \lesssim 10^{6}, T \gtrsim 10^{9}\right)$, where thermal energy is converted into the production of electron-positron pairs, thus resulting in loss of pressure and hydrodynamic instability. This type of supernova was first suggested 40 years ago by Barkat et al. (1967), and since then several works were carried out (e.g. Fraley 1968; Ober et al. 1983; El Eid et al. 1983; Bond et al. 1984; Heger \& Woosley 2002; Hirschi et al. 2004; Eldridge \& Tout 2004; Nomoto et al. 2005), however the overall interest in this topic has been relatively small, mainly due to lack of observational data.

It was originally believed that stars massive enough to produce PISN could only be found among population III stars with close to zero metallicity $\left(Z \lesssim 10^{-4}\right)$, and hence only at very high redshift $(z \gtrsim 15)$. More recently Scannapieco et al. (2005) discussed the detectability of PISN at redshift of $z \leqslant 6$, arguing that metal enrichment is a local process, therefore metal-free star-forming pockets may be found at such low redshifts. Langer et al. (2007) introduced the effect of rotation into studying this question concluding that PISN could be produced by slow rotators of metallicity $Z \lesssim Z_{\odot} / 3$ at a rate of one in every $1000 \mathrm{SN}$ in the local universe. Furthermore, Smith et al. (2007) point out, that mass loss rates in the local universe might be much lower than previously thought, so that massive stars might be left with enough mass to become PISN. This conclusion is also supported by Yungelson et al. (2008) who extensively discuss the mass loss rates and fates of VMS. It is interesting to note, that SN 2006gy took place in the nearby Universe. Following the discovery of SN 2006gy, Umeda \& Nomoto (2008) addressed the question of how much ${ }^{56} \mathrm{Ni}$ can be produced in massive CCSN, while Heger \& Woosley (2008) computed the detailed nucleosynthesis in these SNe.

The interest in VMS is further motivated by the discovery of Ultraluminous X-ray Sources (ULX), which can be interpreted as mass-accreting intermediate mass black holes $(\mathrm{IMBH})$ with mass $\sim\left(10^{2}-10^{5}\right) M_{\odot}$. One of the possible scenarios for IMBH 
formation is by VMS formed by stellar mergers in compact globular clusters (see e.g. Yungelson et al. 2008, and references therein). In this context, Nakazato et al. (2006, 2007 ) studied the collapse of massive iron cores with $M \gtrsim 3 M_{\odot}$. In their first paper they treat the fate of stars of mass $\geqslant 300 M_{\odot}$ which reach the photodisintegration temperature $\left(\approx 6 \times 10^{9} \mathrm{~K}\right)$ after undergoing pair instability. The entropy per baryon of these models at photodisintegration is $s>16 k_{B}$ compared with the classical core-collapse SN with $s \sim 1 k_{B}$. In the second paper they aim to bridge this entropy gap, corresponding to core masses of $(3-30) M_{\odot}$ but claim that there is a lack of systematic progenitor models for this range, hence they use synthetic initial models for their calculations.

In this work we focus mostly on the mass range $M \lesssim 80 M_{\odot}$ (He core mass $M_{H e} \lesssim$ $\left.36 M_{\odot}\right)$ immediately below the range which enters the pair instability region, and present a systematic picture of the resulting CCSN progenitors.

\section{Method}

Since the mass loss rates of stars in this range are highly uncertain, (see e.g. discussion by Yungelson et al. 2008), we avoid dealing with this question by following the example of Heger \& Woosley (2002), and modeling the evolution of helium cores. Our helium core initial models are homogeneous polytropes composed entirely of helium and metals, with metallicity $Z \approx 0.015$, in the mass range $(8-160) M_{\odot}$. The models were then evolved to the helium zero age main sequence. In the following we will refer to these models as "He $N$ " where $N$ is the mass of the model. For comparison we evolved also a few models of regular hydrogen stars, beginning from the zero-age main sequence (ZAMS). We will refer to these models as "MN" where $N$ is the mass of the model. All our models have no mass-loss. We argue that as long as the mass loss rate is not so high that it will cut into the He-core, the evolution after the main-sequence phase will be virtually independent of the fate of the hydrogen-rich envelope. We followed the evolution of each model until the star is either completely disrupted (for the PISN case) or Fe begins to photo-disintegrate (for the CCSN case).

We followed the evolution using the Lagrangian one dimensional Tycho evolutionary code version 6.92 (with some modifications), publicly available on the web (the code is described in Young \& Arnett 2005). Convection is treated using the well known mixing length theory (MLT) with the Ledoux criterion. In the MLT formulation of Tycho, the value of the mixing length parameter fit to the Sun is $\alpha_{M L T} \approx 2.1$ (Young \& Arnett 2005), so we used a value of $\alpha_{M L T}=2$ in our calculations. The nuclear reaction rates used by TYCHO are taken from the NON-SMOKER database as described in Rauscher \& Thielemann (2000).

\section{Results and Discussion}

Among the He-core models we computed, those in the mass range $M \leqslant 36 M_{\odot}$ do not reach pair instability and end their lives as CCSN. Fig. 1 shows the evolution of the central density and temperature (left panel) and the density structure of the pre-SN (right panel), at the moment when the central temperature reaches $7 \times 10^{9} \mathrm{~K}$. The two extreme models He8 and He36 are shown, as well as M80 which has a He-core mass similar to the He36 model, and M20 - a typical CCSN progenitor. The left panel also shows two He-core models - He80 and He160 that reach pair instability. Note that the two models He36 and M80 are indeed very close to each other. An example of the composition at the pre-SN stage $\left(T_{c}=7 \times 10^{9} \mathrm{~K}\right)$ is shown in Fig. 2 . The Fe-core mass is $\approx 3 M_{\odot}$, topped by a shell of $\approx 10 M_{\odot}$ of Si-group elements. The size of the Fe-core (defined as 

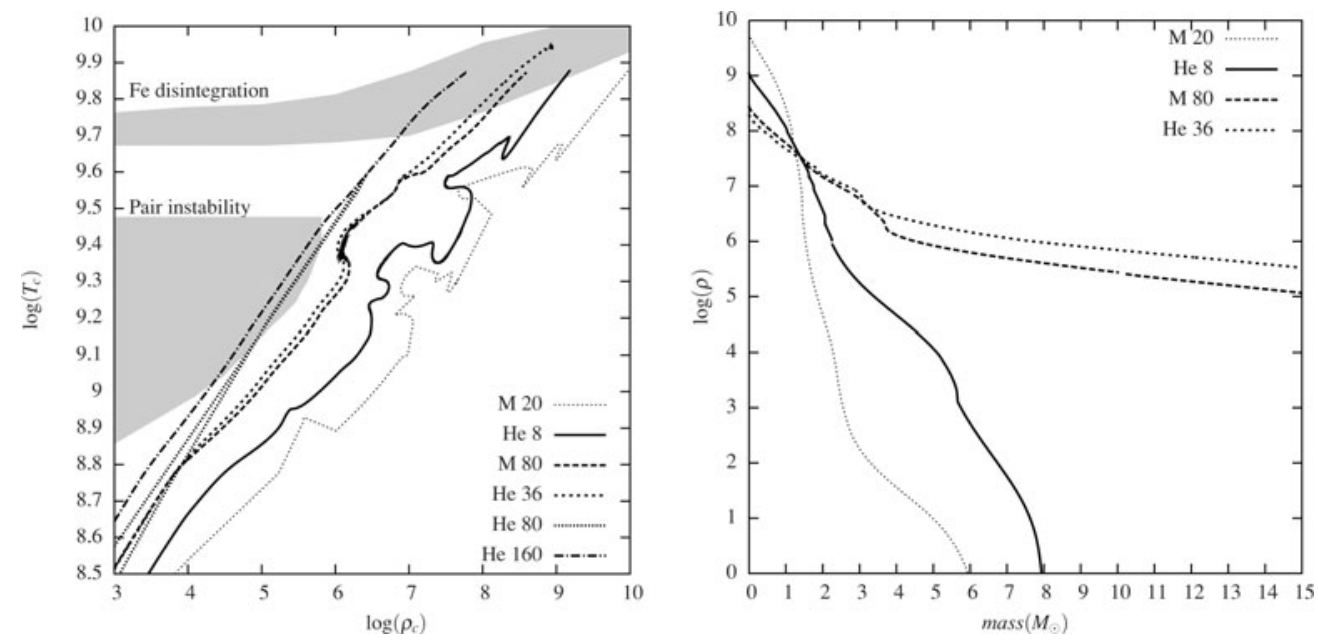

Figure 1. Evolution of the central density and temperature (left panel) and pre-SN density structure (right panel). Each line is labeled "M" for stellar models and "He" for He-core models, followed by the mass of the model.

the mass coordinate where the electron mole fraction $\left.Y_{e}<0.49\right)$ and the central entropy per baryon at the pre-SN stage for the whole set of models is shown in Fig. 3. Note that the size of the Fe-core is slightly non-monotonic. The central entropy, is monotonic with mass, but slightly differs between He-core and stellar models.

As can be seen in the above figures, the outstanding features of these massive models compared with the typical CCSN example M20 are:

(a) Lower central density and higher central entropy.

(b) A much shallower density profile.

(c) Relatively large Fe-cores, up to about $3 M_{\odot}$, and a large amount (up to about $\left.10 M_{\odot}\right)$ of Si-group elements.

These differences might have a considerable impact on the behavior of these models during core collapse and on the outcome of the explosion, a question which we hope to address in the future.

\section{Acknowledgements}

I would like to thank Zalman Barkat for many hours of fruitful discussion. I would like to acknowledge David Arnett for providing his TYCHO code for public use.

\section{References}

Barkat, Z., Rakavy, G., \& Sack, N. 1967, Phys. Rev. Lett., 18, 379

Bond, J. R., Arnett, W. D., \& Carr, B. J. 1984, ApJ, 280, 825

El Eid, M. F., Fricke, K. J., \& Ober, W. W. 1983, A\&A, 119, 54

Eldridge, J. J. \& Tout, C. A. 2004, MNRAS, 353, 87

Fraley, G. S. 1968, Ap\&SS, 2, 96

Heger, A. \& Woosley, S. E. 2002, ApJ, 567, 532

-. 2008, ArXiv e-prints, 803

Hirschi, R., Meynet, G., \& Maeder, A. 2004, A\&A, 425, 649

Langer, N., Norman, C. A., de Koter, A., Vink, J. S., Cantiello, M., \& Yoon, S.-C. 2007, A\&A, 475, L19

Nakazato, K., Sumiyoshi, K., \& Yamada, S. 2006, ApJ, 645, 519

—. 2007, ApJ, 666, 1140 


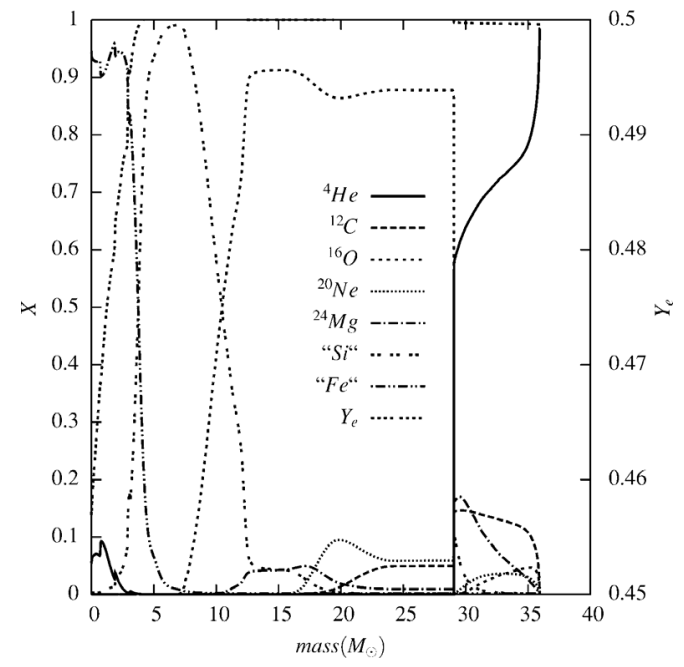

Figure 2. Pre-SN composition of the He36 model. "Si" and "Fe" stand for the total of Siand Fe-group elements respectively.

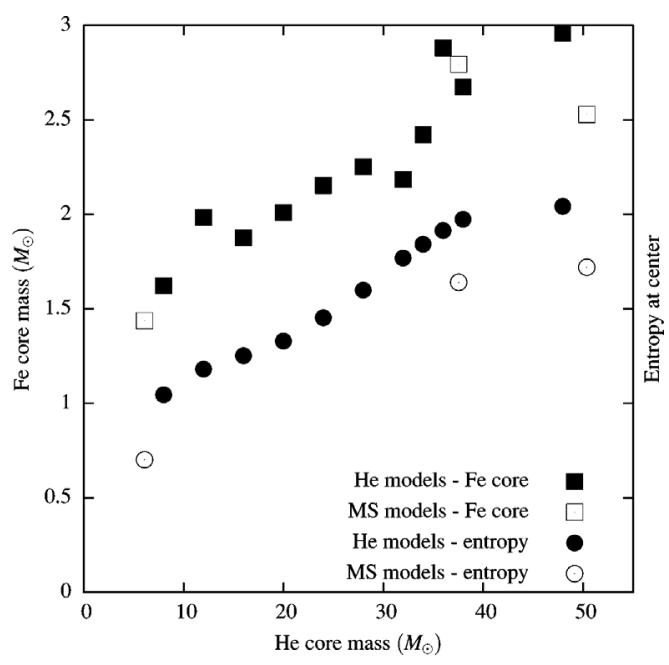

Figure 3. Mass of the Fe-core (squares) and central entropy per baryon (circles) for the computed models. Filled shapes designate $\mathrm{He}^{-}$ core models, open shapes - stellar models.

Nomoto, K., Tominaga, N., Umeda, H., Maeda, K., Ohkubo, T., Deng, J., \& Mazzali, P. A. 2005, in Astronomical Society of the Pacific Conference Series, Vol. 332, The Fate of the Most Massive Stars, ed. R. Humphreys \& K. Stanek, 374-+

Ober, W. W., El Eid, M. F., \& Fricke, K. J. 1983, A\&A, 119, 61

Rauscher, T. \& Thielemann, F.-K. 2000, Atomic Data and Nuclear Data Tables, 75, 1

Scannapieco, E., Madau, P., Woosley, S., Heger, A., \& Ferrara, A. 2005, ApJ, 633, 1031

Smith, N., Li, W., Foley, R. J., Wheeler, J. C., Pooley, D., Chornock, R., Filippenko, A. V., Silverman, J. M., Quimby, R., Bloom, J. S., \& Hansen, C. 2007, ApJ, 666, 1116

Umeda, H. \& Nomoto, K. 2008, ApJ, 673, 1014

Woosley, S. E., Blinnikov, S., \& Heger, A. 2007, Nature, 450, 390

Young, P. A. \& Arnett, D. 2005, ApJ, 618, 908

Yungelson, L. R., van den Heuvel, E. P. J., Vink, J. S., Portegies Zwart, S. F., \& de Koter, A. 2008, A\&A, 477, 223

\section{Discussion}

KUPKA: Are there any observations available for the candidate supernovae you have mentioned in your talk, which would support the element yield rates your progenitor models would predict? Since their interior structure is quite different from the usual corecollapse progenitor models, one would expect also a distinctive pattern in abundances (mass fractions) produced by the subsequent supernovae.

WALDMAN: As far as I know there is no conclusive abundance data that can affirm the identification of these SNe with PISN. 